\title{
The Use of the Nintendo Wii in Motor Rehabilitation for Virtual Reality Interventions: A Literature Review
}

Dr Emmanuel Tsekleves ${ }^{1}$, Mrs Alyson Warland ${ }^{2}$, Dr Cherry Kilbride ${ }^{2}$, MrIoannis Paraskevopoulos ${ }^{3}$, DrDionysios Skordoulis ${ }^{3}$

\begin{abstract}
Several review articles have been published on the use of Virtual Reality (VR) in motor rehabilitation. The majority of these focus on the effectiveness of VR on improving motor function using relatively expensive commercial tools and technologies including robotics, cybergloves, cybergrasps, joysticks, force sensors and motion capture systems. However, we present the case in this chapter that game sensors and VR technologies which can be customized and reconfigured,such as the Nintendo Wii, provide an alternative and affordable VR intervention for rehabilitation. While the performance of many of the Wii based interventions in motor rehabilitation are currently the focus of investigation by researchers, an extensive and holistic discussion on this subject does not yet exist. As such, the purpose of this chapter is to provide readers with an understanding of the advantages and limitations of the Nintendo Wii game sensor device (and its associated accessories) for motor rehabilitation and in addition, to outlinethe potential for incorporating these into clinical interventions for the benefit of patients and therapists.
\end{abstract}

Keywords:Nintendo Wii, motor rehabilitation, literature review, virtual reality, gaming

\footnotetext{
${ }^{1}$ ImaginationLancaster, Lancaster University, LICA Builiding, Bailrig, Lancaster, LA1 4YW, UK.e.tsekleves@lancaster.ac.uk (corresponding Author)

${ }^{2}$ Centre for Research in Rehabilitation, Brunel University, Kingston Lane, Uxbridge, UB8 3PH, UK. alyson.warland@brunel.ac.uk, cherry.kilbride@brunel.ac.uk

${ }^{3}$ School of Engineering and Design, Brunel University, Kingston Lane, Uxbridge, UB8 3PH, UK.Ioannis.Paraskevopoulos@brunel.ac.uk, Dionysios.Skordoulis@brunel.ac.uk
} 


\section{Introduction}

Recently,a number of studies have examined the use of virtual reality (VR) in motor rehabilitation(Ma and Bechkoum 2008). These have employed a variety of VR technologies, such as robotics, cybergloves/cybergrasps, joysticks, force sensors and motion capture systems(e.g. IREX, PHANToM; SensAble Technologies Inc). The majority of studies have focused on the use of VR in the rehabilitation of neurological disorders (Rahman \& Shaheen 2011; Saposnik \& Levin 2011); in particular, as an adjunct to promote upper limb recovery after stroke (Brochard et al. 2010; Henderson et al. 2007; Laver et al. 2011). However, VR has also been utilized in the wider arena of rehabilitation including, rehabilitation of older people(Kwok et al. 2011), balance (Crotty et al. 2011; Gil-Gómez et al. 2011), burns (Fung et al. 2010), cardiovascular and general fitness (Nitz et al. 2010), amputee rehabilitation (Oppenheim 2010), orthopedics (Fung et al. 2012), pediatrics (Wuang et al. 2011) and intensive care (Kho et al. 2012).

Review papers concluded that while novel, VR and video game technologies could potentially be beneficial in rehabilitation and could be combined with conventional therapy(Rahman \& Shaheen 2011; Saposnik \& Levin 2011; Brochard et al. 2010). However, only a limited number of studies included in the reviews, focused on commercial VR gaming systems, such as the Nintendo Wii (Rahman \& Shaheen 2011; Saposnik \& Levin 2011).

Use of gaming in rehabilitation is gaining in popularity and fits with current theories of motor recovery and motor learning which highlight the need for therapy to be motivational (Saposnik et al. 2010b), intense and repetitive in order to maximize recovery (Langhorne et al. 2009); The Nintendo Wii stands at the forefront of this development and is based around an intuitive motion sensor system that provides real-time information on performance, using visual, auditory and sensory feedback. However, the Nintendo Wii (as with other gaming sensor devices) has been designed for "fit and healthy" individuals, leading to concerns about its suitability for rehabilitation purposes. As a result, a number of researchers have evaluated the bespoking of such technologies(Alankus et al. 2010; GilGómez et al. 2011; Lange et al. 2010; Milosevic and Jovanov 2011; Van Wieringen and Eklund 2008), yet to date, there are no literaturereviews in this growing field of investigation.

This review has been conducted by a multidisciplinary team of computer engineers, designers and physiotherapists, with the purposeof:

- Defining the technical characteristics of the Nintendo Wii remote and balance board (section 2), in order to facilitate the discussion of the technical merits and limitations of the technology.

- $\quad$ Reviewing the evidence for how the Nintendo Wii is being used and evaluated as a clinical adjunct to motor rehabilitation (section 3 de- 
scribing review methodology and section 4 outlining the main findings)

- Detailing the advantages and limitations of the Nintendo Wii in motor rehabilitation, including any reports of adverse effects (section 5.1 and 5.2)

- Highlighting potential uses of the Nintendo Wii in motor rehabilitation (section 5.3)

- $\quad$ Identifying areas for future research (section 5.3.1).

\section{Technical Characteristics of the Nintendo Wii}

\subsection{The Nintendo Wii Remote (Wiimote)}

The Wii Remote is a wireless low-cost controller for the Nintendo's Wii console that allows the user to interact with games and applications via gesture recognition. In electronics, the Wiimote is known as an inertial measurement unit (IMU) as it includes a combination of accelerometers and gyroscopesthat measure velocity, orientation and gravitational forces.

Degrees of Freedom and Frame of Reference. The position of a rigid body has two components: linear and angular. A linear position displacement occurs at three perpendicular axes of the body's frame of reference (FoR) relative to another FoR, such as the earth's. Combined with angular displacement (rotation) on its own reference frame, this can provide the motion information of that body in threedimensional (3D) space(Laviola et al. 2010). In other words, it can place the Wii remote in a $3 \mathrm{D}$ space environment. This motion is usually referred to as, forward/backward, up/down, left/right for the linear position and yaw, pitch and roll for the rotation. As the movement along each of the three axes is independent of each other and independent of the rotation about any of these axes, the motion is classified as having six degrees of freedom (6DoF) (Featherstone 1987).

Connection with the Computer.The Wiimote typically communicates with a Nintendo Wii game console wirelessly via the Bluetooth interface. Equally, it can be paired with any device that supports the Bluetooth stack which implements the Bluetooth Human Interface Device (HID) protocol, such as a personal computer or laptop. Once the Wiimote is in a "discovery" mode (buttons ' 1 ' \& ' 2 ' pressed simultaneously or the red sync button at the back) the operating system (OS) will recognize a HID-compliant device and a connection will be established. Considerable effort by the open source community has resulted in the ability to reverse engineer the structure definitions of the input stream, functions and signature calls. 
Various Wii-orientated, open source libraries have facilitated the interaction with a computer by using a set of common library functions:

- WiiYourself!(gl.tter 2013)- A Windows OS based code, implemented in $\mathrm{C} / \mathrm{C}++$, fully-featured for most Wii's accessories (including Wii Motion Plus, Balance Board, Nunchuck and etc.) and supports all Bluetooth stacks.

- GlovePIE(Kenner 2010)- is a free Windows Programmable Input Emulator that uses scripts to interact with the computer. It is bounded on the functions provided by the emulator and no adaptation is possible since it is not distributed with the source code. Nevertheless, it is easy to use and provides an adequate support to most of the accessories.

- WiimoteLib(Peek 2010)- A library for using a Nintendo Wii Remote from .NET. Recentlyupdated for supporting Wii MotionPlus (the integrated version of the Wiimote and the Wii motion plus accessory). However, it was stated by the author as incomplete.

- Wiiuse(Laforest 2010)- A cross-platform C library that can be used with $\mathrm{C} / \mathrm{C}++$, Java and Python. It is light (in terms of the processing demands) and has a well-documented application programming interface (API). For Wii MotionPlus support, reference to other extensions from this library is required (e.g. libogc).

- CWiid(Smith 2011)- A collection of Linux tools written in C for interfacing to the Nintendo Wiimote.

The choice of the library depends entirely on the system requirements and operating platform. The Wiimote includes an accelerometer and a gyroscope device, which are discussed below.

Accelerometer. The remote has the ability to sense acceleration along three axes via a three-axis linear integrated chipset ${ }^{1}$. Its main physical characteristic is that it can acquire measurements over a range of at least $3 \mathrm{~g}$ (gravity force) with $10 \%$ sensitivity. Since the accelerometer actually measures the force wielded by a set of small proof masses inside the circuit with respect to its enclosure, it can only track liner gravity acceleration $g$ (approximately $-9.8 \mathrm{~m} / \mathrm{sec} 2$ ) in a free fall frame of reference and not horizontal rotation. Therefore, it can only be used to derive pitch and roll from the respective axes but not the yaw orientation(Seifert and Camacho 2007). Pure accelerometer-based solutions can only be deployed for applications with a fixed reference from gravity as well as linear or tilt movement, constrained to limited rotation.

Gyroscope. Gyroscopes can measure angular velocity which may be integrated over time to compute the sensor's orientation directly. Based on the principles of conservation of angular momentum, when the gyroscope device is rotated, the Coriolis force creates an orthogonal vibration force proportional to the rate of rota-

\footnotetext{
${ }^{1}$ Analog Devices, Inc., "ADXL330 Data Sheet Rev A,” September 2009. Available: http://www.analog.com/static/imported-files/data_sheets/ADXL330.pdf [6 November 2013].
} 
tion. The Wii MotionPlus is the gyroscope component of the controller and comprises dual-axis and single-axis integrated gyroscope sensors. The first version of Wii MotionPlusfeatured an external peripheral attached at the bottom-end of Wii's remote controller ${ }^{2}$. As it features both dual-axis (for pitch and roll angular velocities) and single-axis (for yaw angular velocity), it has the capability to report full orientation tracking. Similarly with the accelerometer, the gyroscope's readings are in raw binary form (using a 14-bit ADC module) in orderto get the actual angular velocity expressed in degrees per second.

\subsection{The Nintendo Balance Board}

The Wii Balance Board is a game controller that is predominantly used in combination with the Wii and its associated software. It uses standard Bluetooth technology to communicate with the Wii. In addition it can be connected to a personal computer using any of the aforementioned open source software applications. The balance board allows players to control games simply by shifting their weight. This is achieved by four pressure sensors situated at each corner of the board (with $43 \mathrm{~cm}$ between left and right sensors and $21 \mathrm{~cm}$ between top and bottom sensors) allowing the detection of slight movements and determining foot position and weight distribution. Each sensor can assume a weight value, which is equivalent to $34 \mathrm{~kg}$.

\section{Review of Literature}

\subsection{Search Methodology}

The search strategy was developed in stages. An initial text search of ACM (Association for Computing Machinery) and IEEE (Institute of Electrical and Electronics Engineers) was carried out using the terms "Nintendo Wii AND motor rehabilitation OR Neurorehabilitation"to find exemplar articles from which to harvest indexing terms. The bibliographic details for each item from the initial search were reviewed independently by members of the project team (ET and DS).

Following this, the categories and keywords were fine-tuned to ensure all exemplar articles were returned in the final database search. An extensive literature

\footnotetext{
${ }^{2}$ InvenSense Inc., "World's Leading Multi-axis MEMS Rate Gyroscope Enhances Performance of Latest Wii MotionPlus Accessory" May 2010. Available: http://invensense.com/mems/gyro/documents/articles/071508.html [6 November 2013].
} 
search was conducted using the seven electronic research databases most frequently used in the fields of technology, computer science, engineering and medicine: the IEEE Explore,ACM, Science Direct, Web of Science, Scopus, MEDLINE and PubMed.

The search was performed in January 2012using the following keywords: Nintendo Wii, Wii remote, Wiimote, Wii balance board, Nintendo balance board, Wii fit, rehabilitation, motor rehabilitation, neurorehabilitation, stroke. These keywords were combined using the Boolean operator AND or OR with the aforementioned keywords to create the following three groups of search terms:

(Nintendo Wii OR Wii remote OR Wiimote OR Wii balance board OR Nintendo balance board OR Wii fit) AND (rehabilitation OR motor rehabilitation OR neurorehabilitation OR stroke)

Search terms were entered in each selected database, providing a unique search outcome per database (see Fig 1).

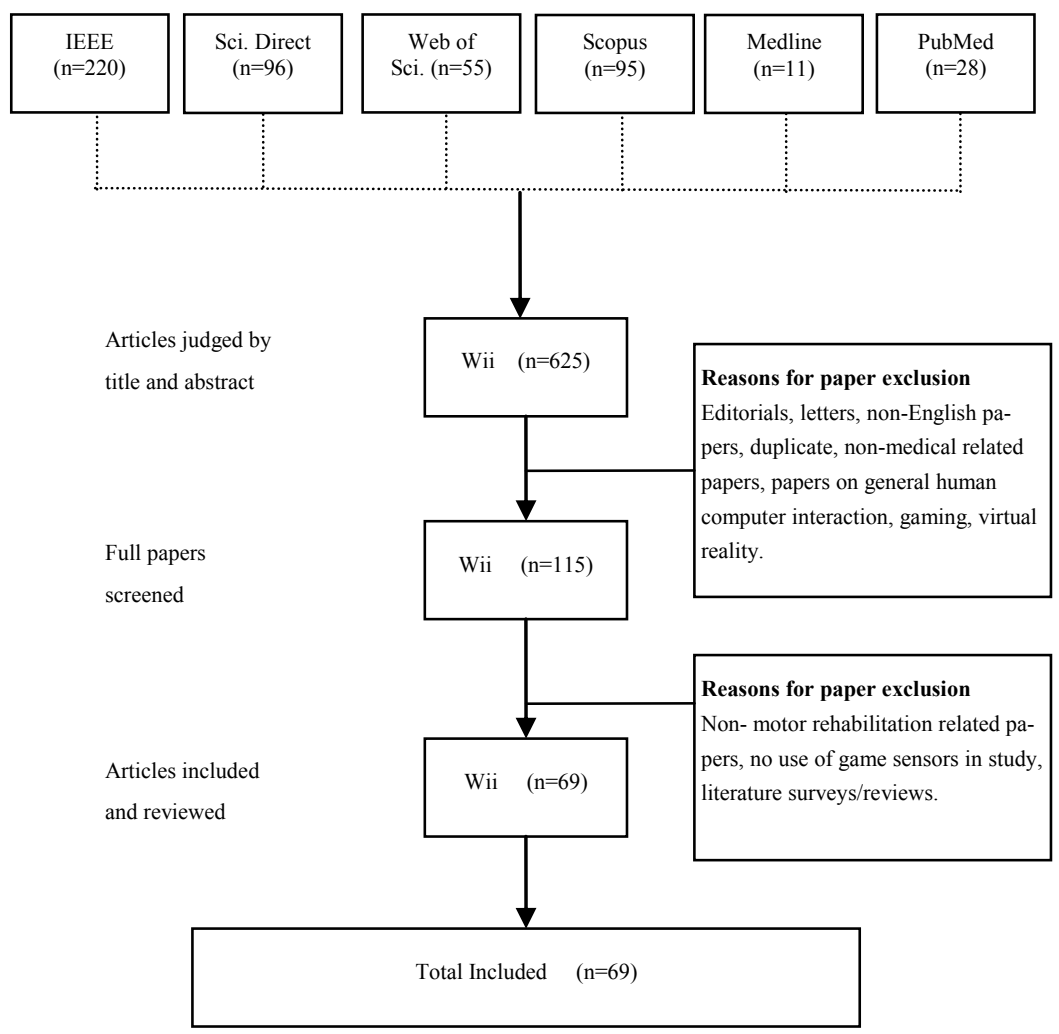


Fig 1. Search Strategy.

Article titles and abstracts were read (by ET and DS) and those that matched the review questions, inclusion/exclusion criteria and keywords were retrieved. In the case that the title and abstract did not contain enough information to decide on inclusion, the full article was read. Further screening of the full papers was performed by the review team (ET, DS, CK, IP, AW). Papers retained for the final review were based on the original review questions as well as a second set of inclusion/exclusion criteria (see Fig 1). All papers were screened by two reviewers independently. Discrepancies were resolved by consensus or involvement of a third reviewer.

Inclusion and Exclusion Criteria: Inclusion and exclusion criteria were used to refine the search results. Firstly the search was limited to English text papers published in peer-reviewed journals and conference proceedings from January 2006 (when the Nintendo Wii was released) to January 2012. Secondly, editorials, letters, book reviews and book chapters were excluded, as were papersunrelated to motor rehabilitation and those that did not employ the use of game console sensor devices. In addition, articles which related solely to the use of the game console sensor devices for the purposes of human computer interaction (HCI), gaming and VR in general, were excluded from the study. Literature review papers were not included in the final critical appraisal but were drawn upon for cross-validation of our findings.

Figure 1 shows 625 original articles and reviews were identified. Inclusion and exclusion criteria (as outlined above) were applied to the abstracts resulting in the removal of 510 articles. The remaining 115 full text articles were scrutinized and a further 46 were excluded at this stage, resulting in the inclusion of 69 original articles for this review.

\section{Summary Findings}

Study characteristics are presented in (see externally linked file: Literature Review Summary Table). All papers discussed the efficacy and/or development of the Nintendo Wii for motor rehabilitation. These can be divided into five key areas, namely:

- game sensor device type (i.e. the Nintendo Wii remote and/or balance board);

- level of sensor device customization (i.e. whether the console was original or customized (bespoke) for the purposes of the specific intervention); 
- type of intervention (i.e. whether a console game was used, custom made game or custom made exercise was developed);

- $\quad$ study characteristics (i.e. specific characteristics of the intervention such asthe study design, duration, participant population and outcome measures used) and finally;

- the study results (i.e. intervention effect and whether any adverse eventshad occurred).

Game Sensor Device Type. Sixty-nine studies employed the Nintendo Wii remote sensor and/or balance boardas a tool for motor rehabilitation. The remote sensor device was more widely employed, being specifically used in 38 studies (55.1\%) compared to $25(36.2 \%)$ which only used the balance board and $6(8.7 \%)$ which included both devices. The reason behind this can be sought in the specific health specialties where each sensor has been used. For example the Wii remote has been employed by 20 studies developing interventions for upper-limb stroke rehabilitation and other upper limb motor rehabilitation pathologies, such as orthopedics, amputee rehabilitation, cerebral palsy. The balance board, on the other hand, has been utilized mainly for balance specific disorders.

Level of Device Customization.Closer scrutiny of the Nintendo Wii game sensor studies revealed that overall36 studies $^{3}(52 \%)$ employedcustomized versions of either the Wii remote or balance board. Customization of the Wii game sensors suggests that instead of using the commercially available Nintendo Wii console for rehabilitation, the authors usedbespoke versions where the Wii game sensors were connected to a personal computer (PC). It is interesting to note thatgame sensor bespokingoccurred in $67 \%$ of studies thatemployedthe Wii remote sensor (30 out of 45 studies) of which $50 \%$ were in neurological conditions, whereas only $32 \%$ of studies (10 out of 31 ) bespoke the balance board. This indicates that for the purposes of motor rehabilitation, theWii remote requires customization more often than thebalance board in particular for use by neurological patients.

Type of Intervention.Despite the tendency for game sensor customization, there was a balance between the use of standard commercially available console games and the development of custom games and exercises. Thirty-five studies used existing games played on the corresponding game console and 34utilizedeither custom developed games (13 studies) or custom developed exercises (21 studies)to help deliver motor rehabilitation.

With regard to the customization of the intervention, two distinct groups were apparent: those which developed customized exercises and those which developed customized games.In the former, the patient accomplished tasks in the context of a game, whereas in the latter, the patient was directly guided throughout the move-

\footnotetext{
${ }^{3}$ Two of the 36 studies employed customization for both the Wii remoteand balance board
} 
ment.Typically VR environments for upper limb rehabilitation were categorized as game-like or teacher-animation(Lehrer et al. 2011).

Customized exercises weretypically therapist-designed and requiredrepetition ofdesired movements, whereas the customized games involved game design concepts and game mechanics for conducting rehabilitation exercises in a more motivating and implicit manner(Annema et al. 2010; Ballaz and Robert 2011; Burke et al. 2008; Gerling et al. 2010; Geurts and Abeele 2011; Gil-Gómez et al. 2011; Göbel et al. 2010; Jung et al. 2009; Kho et al. 2012; Lange et al. 2011; Levac et al. 2010; Robinson and Schaik 2011; Saposnik et al. 2010a; Williams et al. 2011). Moreover, both weredesigned to be used on a PC instead of the original game console device.

Study Characteristics. The Wii was usedacross a variety of clinical specialties such as neurology (including stroke), rehabilitation of older people, respiratory, burns, pediatrics, intensive care, amputees, falls \& balance and orthopedics, suggesting that the Wii may potentially be a useful adjunct in the rehabilitation of a wide variety of conditions. Stroke wasthe most commonly explored condition ( $43 \%$ - 30 studies), followed by balance disorders (22\% - 15 studies) and then other motor rehabilitation ( $12 \%$ - 8 studies). In studies that only used the Wii balance board sensor, 58\% (18 of 31 studies) explored balance in general and 42\% (13out of 31 studies) looked at balance specifically in stroke.

The review revealed that the Wii is being used as an assessment device and for treatment. Of the studies reviewed, $9(13 \%)$ were concerned with using the Wii as an assessment tool and $60(87 \%)$ were concerned with using the Wii as a treatment. Overall, 34(49\%) looked at the efficacy of using the Wii as a treatment, 30 $(43 \%)$ were more focused on development of Wii as a treatment (including feasibility, acceptability and technical details regarding customization) and 5 (7\%) looked at both aspects)..

Methodological issues limit the conclusions that can be drawn as the majorityof studies $(n=31,45 \%)$ were pilot or quasi-experimental (utilizing either prepost designs, or pilot studies, or single case experimental design, or controlled trials without randomization). Only 9 (13\%)wererandomized controlled trials (RCT). Five studies (7\%) were observational. A further 5 were case studies (7\%) and 4 involved qualitative methodologies $(6 \%)$. Many devices appeared to be untested $(\mathrm{n}=16,23 \%)$ whereas others were tested but on a non-target group (e.g. a device designed for use in neurology was tested on people who were neurologically intact). In addition, the majority of studies were underpowered withsmall sample sizes.

Furthermore there was a lack of information regarding the duration of the study in $22(32 \%)$ of the 69 studies. Where stated, the most common study duration was 2 weeks ( $\mathrm{n}=20,29 \%)$.Threestudies lasted for 5 weeks, 2 for 12 weeks and 2 for over 3 months. In terms of the duration of the intervention,31 (45\%)) lasted 
for one hour, $14(20 \%)$ lasted for 20 minutes, 14 (20\%) lasted for 30 minutes, 7 $(10 \%)$ lasted 10 minutes and $4(5 \%)$ lasted for 15 minutes. Interestingly only fourstudies involving stroke (Alankus \& Proffitt 2010; Gil-Gómez et al. 2011; Mouawad \& Doust 2011; Saposnik et al 2010;) achieved the UK recommended minimal intensity of 45 minutes a day andnot all of these managed to deliver the intervention for the recommended five days (National Institute for Health and Clinical Excellence 2013).

As can be seen from the externally linked Table (see Literature Review Summary Table)avariety of outcome measures were employed. These included measures of impairment of body structure and function (such as the Modified Ashworth Scale and Fugl-Meyer Assessment), measures of activities (such as the Berg Balance Test, 10 Meter Timed Walk) and measures of participation (such as the Stroke Impact Scale). Few studies employed measures looking at all three levels. In addition, a number of studies employed outcomes, which were not validated or tested for reliability and others suffered from the use of measures which were not sufficiently sensitive to detect clinical change and this may have affected the results obtained.

Study Results. There is increasing evidence in support of the use of the Wii for rehabilitation in a number of different clinical areas including the upper limb following stroke(Manlapaz et al. 2010; Mouawad and Doust 2011; Saposnik et al. 2010a; Yong Joo et al. 2010), balance(Bateni 2012; Crotty et al. 2011; Deutsch et al. 2009; Gil-Gómez et al. 2011), post total knee replacement (Fung et al. 2012) and fitness (Ballaz and Robert 2011; Hurkmans et al. 2011; Morelli et al. 2011). However, the evidence is not conclusive and more robust studiesare required (incorporating changes suggested in the next sections of this paper) before the technology can be integrated into rehabilitation outside of the research setting.

Relatively few adverse effects were noted suggesting that the Wii is a safe to use with subjects with motor problems. When reported, adverse effects were minor in nature, such as mild shoulder pain (reported by 2 out of 10 stroke patients following 30 minutes of intense exercise)(Hurkmans et al. 2011), knee pain (one person with multiple sclerosis)(Plow and Finlayson 2012), slight loss of balance stepping off the balance board and one episode of light-headedness on exercising with the Wii (Williams et al. 2010). Interestingly, no falls werereported in any study and no adverse effects (including cardiovascular issues or accidental removal of artifacts i.e. intravenous lines) were reported when using the Wii with 22 subjects in an intensive care setting (Kho et al. 2012). 


\section{Advantages and Limitations of the Nintendo Wii in Rehabilitation}

This section brings together findings from the literature review related to the key advantages and limitations of the using the Nintendo Wii in motor rehabilitation from both the technical and clinical perspective.

\subsection{Technical Advantages and Limitations}

A number of technical advantages and limitations have been highlighted from the literature.

The Nintendo Wii is a relatively affordable gaming console (around $£ 180^{4}$ ) with the accessories of the balance board andWii remote also available at comparatively accessible costs of around $£ 80$ and $£ 35$ respectively. It is a relatively affordable piece of equipment that is commonly found in many homes and therefore.specific exercises (from the Wii sports and fitness game series) and game challenges can be given to people to perform at home as part of their homerehabilitation program.

As the Nintendo Wii accessories can be purchased individually,they can be used with a personal computer (using the available open source software applications presented in section 2) to create customizedapplications. The open source software applications, although free to use, require considerable technical knowledge to be employed. However, theyallow up to four Wii remotes to be simultaneously connected to a computer at a given time. It is therefore possible to create games or exercise routineswhere the Wii remotes can be strapped (using arm/leg bands) to the arms or legs of the user to drive the games or rehabilitation exercises. Bluetooth compatibility issues with different types of stack and various versions of the Wiimote, means that at times, the process of pairing a Wii remote with the computer has to be done manually,something most users can be easily trained to do. An alternative is to use commercially available Bluetooth pairing software such as the BlueSoleil Software ${ }^{5}$.

As can be seen in the summary findings section (section 4), half of the studies examined have opted for customizing the Wii. The main reasons for thisare as follows:

- Accessories such as the Wii remote and balance board provide cheap IMU and balance training equipment when compared to similar commercially available

\footnotetext{
${ }^{4}$ See Amazon UK at www.amazon.co.uk, prices in June 2013

${ }^{5}$ BlueSoleil Software. Available at: http://www.bluesoleil.com/index.aspx[6November 2013]
} 
therapy solutions, such as high-cost force plate systems or optical motion capture systems(Bainbridge et al. 2011; Galego \&Simone 2007; Huber et al. 2010; Jovanov et al. 2009; Milosevic \& Jovanov 2011).

- The data generated from the commercially available Wii remote and balance board when used with the Nintendo Wii console cannot be extracted for analysis by the therapist. For instance, valuable information such as the amount of acceleration, the degree and range of movement can only be accessed via pairing the Wii remote or balance board with a computer using the open source software available.

- The unsuitability of many of the commercially available game packages limits their potential use to just a few health conditions, such as musculoskeletal. Games ideally should be able to be customized and personalized for the individual and their health condition in terms of the level of difficulty, the speed of gameplay, the range of motion, and types of exercises.

- Although the interaction paradigm of engaging with the games through physical interaction is generally regarded as intuitive, issues are apparent when using commercial games and exercises for the purpose of motor rehabilitation (Jung et al. 2009). For example, feedback has beenidentified as inappropriate and often discouraging by users with motor disabilities(Anderson et al. 2010; Deutsch et al 2009, Huber et al. 2010; Sugarman et al. 2009) and scoring systems have been found inadequate as a measure of performance and progress (Burke et al. 2009; Lange et al. 2010; Sugarman et al. 2009).Furthermore,the amount of time to set up the equipment has been identified as an issue for therapists with Annema et al (2010) noting this frequently took up half of the treatment time and Mouawad\&Doust (2011) observing that stroke patients frequently required over ten minutes to activate games compared to less than one minute for the neurologically intact .

- Monitoring of patient performanceis an essentialelement for therapists, who unless physically present cannot evaluate and monitor patient performance and adherence to exercise (Egglestone et al. 2009; Martin-Moreno et al. 2008; Prashun et al. 2010; Saposnik \& Levin 2011). Logging of data and patient performance is only possible through employing a customized Wii solution.

- The accuracy of motion and balance data,results in the capture of more robust and clinically important data, such as range of movement across three or more DoF.

For data accuracy, it should be noted thatalthough the Wiimote includes both an accelerometer and a gyroscope, acquiring an accurate 3 DoF movementknown as pitch, roll and yaw from the device itself is very challenging. For this reason the Nintendo Wii console comes with an LED sensor bar that the infrared sensor positioned at the front of the Wii remote employs to detect the yaw movement of the remote. However this only works provided the two devices are in line of sight (facing each other directly over a specific distance). This limits the type of exercises and range of movement that can be achieved. For instance exercises which 
require a large range of motion (such as a basic shoulder flexion-extension movement)cannot be captured by the Wiiremotes (Alankus et al. 2010).

In addition to this, as electromechanical devices, both the accelerometer and gyroscope, are prone to noise, errors and bias( Jovanov et al. 2009). This can be minimized, thereby improving the accuracy of the Wiimote, by filtering these out. However, it is important to avoid degradation of the raw output data while doing this. There are various methods to filter out the noise, someinvolve complex computations but offer impressive results and others have few computational methods but use data that is initially very distorted(Meditch 1973). The choice of selecting the right trade-off depends on the characteristics of the signal (peak position, height, width, area, etc.) and system requirements. Itshould be noted that in most algorithms, the intervals between adjacent data points or within a window frame must be obtained at the same time. Both theWiimote's accelerometer and gyroscope have a sampling rate of $100 \mathrm{~Hz}$ but in reality there is a uniform distributed delay of 1 to 5 milliseconds, which is considered to be a negligible part of the computer's processing cycle.

Additional issues can be found with the data produced by the gyroscope, which calculates the yaw range of motion. After significant rotation the yaw orientation will be misaligned, also known as "drift", because IMUs suffer from accumulated error; they continually add detected changes to their previously-calculated positions and any errors in measurement, however small, are accumulated from point to point. Thus, the yaw position has to be reset to a known point (e.g. zero as the pointing forwards) otherwise the yaw rotation given will be inaccurate.

Several studies have developed customized Wii-based interventions aimed at developing a VR-based system that captures the patient's armmotion in a more accurate way compared to the conventional Wii remote. These can be categorized into three main groups depending on the technical methodology employed for customizing the Wii remote: acceleration data only, Wiimote and light-emitting diodes (LEDs) as an infra-red (IR) camera and a hybrid of the two.

The majority of customizedWii remote stroke interventions employ one or twoWiimotes with a reverse engineered(API) to capture the patient motion by reading the Wiimote accelerometer data.

A number of algorithms have been suggested and implemented by various research groups to filter dataand map them to motion, in order to drive either a set of exercises or custom-built games (such asLeder \& Azcarate 2008; Van Wieringen \& Eklund 2008; Alankus et al. 2010; Matamoros \& Negrete 2010). Since all these approaches obtain the position of the Wiimote in space using the acceleration data (that is the change inthe linear acceleration as the patient moves the Wii remote in space) they suffer from a DoFlimitation. More precisely, these solutions offer accuracy only in 2-DoF as the acceleration data can only determinethe pitch and roll movement. Such systems are therefore more appropriate for gesture-based inter- 
ventions ratherthan one-to-one mapping (kinematic animation) of movement onto the VR environment.

Another common Wiimotecustomization involves using a pair of Wiimotes with the LED sensor bar or customLEDs to act as an IR camera to create a lowcost motion capture system(Jovanov et al. 2009; Milosevic \& Jovanov 2011; Attygalle \& Duff 2008; Scherfgen \& Herpers 2009; Martin-Moreno et al. 2008; Wilson et al. 2007). Reflective markers or LEDs areusually placed on patient's arm or hand and as the exercises are executed, the range of motion is captured andmapped onto the system display. The limitation in this approach is that each Wiimote can only detect up to fourLEDs in space, thus restricting the range of movement and set of exercises that the patient can do. Caremust also be taken to ensure the angle of the Wiimotes does not obstruct the LEDs as the patient moveshis/her arm. Moreover, the use of LEDs often requires extra power supply devices to be attached to patients.Wilson(Wilson et al. 2007) and MartinMoreno(Martin-Moreno et al. 2008)have proposed a hybrid solution that incorporates the two aforementionedapproaches in an effort to increase accuracy and the number of DoFs. However, this approach also suffers from alimited field of view by employing the Wiimote as an IR camera making it unsuitable for tracking larger motionsoften required in therapy(Alankus et al. 2010; Geurts et al 2011).

Although not designed specifically for motor rehabilitation, Williamson et al(Williamson et al. 2010)have proposed a Wii-based system that merges accelerationwith gyroscope data and the Wiimote's IR. This system is aimed at motion recognition for sport-like games (e.g.American football) and not for kinematic analysis and mapping of user motion ontoa 3D avatar (virtual character) but could potentially be adapted for motor rehabilitation. Their developed data fusion algorithm requires the use of the Wiimote's IR to compensate for the gyroscope drift and movement corrections. Results indicate improved motion recognition when compared to acceleration data alone but still show loss of accuracy when the IR is out of sight.

There are other technical solutions that could resolve the yaw orientation drift issue and hence provide a fully accurate $3 \mathrm{DoF}$ Wii remote system without the need to use the LED sensor bar that comes with the Nintendo Wii console.One solution is to integrate a magnetometer device. Magnetometers are electromechanical devices, thatmeasure the strength of direction of the earth's magnetic fields and can be used as a reference point for correcting the gyroscope's yaw drift.

Another workable approach to the problem is to process concurrently and conjointly both sensors' (accelerometer and gyroscope) measurements through a data fusion algorithm(Tsekleves et al. 2011). There are a number of different fusion algorithms that can be employed, such as the discrete-time complementary Kalman filter (Kalman 1960), the Newton-Raphson optimization method (Madgwick 2010) and several others. The decision on which to use is a trade-off between the computational power available and the accuracy level required for theWii system. 
For instance, higher accuracy levels can be achieved using the Kalman filter but a powerful computer will be required to process the data fusion algorithm and the data in real-time.

Raw information captured from one sensor can be used to distinguish true readings of the other, and vice versa. In addition, the angular rate measurements captured by the gyroscope sensor can be used to distinguish true linear motion from the accelerometer readings. When used in combination with a matching human body's kinematic analysis, a highly accurate, highly responsive, one-to-one representation of the control device in 3D space (with $3 \mathrm{DoF}$ ) can be achieved.

An additional advantage of the Wii remote, currently underutilized by the research community, is the possibility for providing the patient with haptic feedback via its rumble feature (Martin-Moreno et al. 2008; Morelli et al 2010b).

Compared to the Wii remote, the balance board is easier to customize and incorporate from a technical point of view, although knowledge of how to use the open source software applications presented in section 2 is still required. By knowing the location and distance of the four sensors, a number of different algorithms can be used, to calculate with accuracy the weight transferand position of the person on the board(Kennedy et al. 2011; Gil-Gómez et al. 2011; Clark et al. 2010). For instance, different movements such as a jump, press up or knee bend can be identified, displayed graphically on a $3 \mathrm{D}$ character on the computer screen and recorded for later monitoring by the therapist. These movements can either be incorporated into a game or given to patients as exercises to follow. A key advantage of the balance board is that it can be used as an affordable alternative to more expensive force platforms(Bainbridge et al. 2011; Bateni 2012; Clark et al. 2010). As with the Wii remote, patients using the balance board do not explicitly associate the exercises with rehabilitation but with gaming and fun (Gil-Gómez et al. 2011). In addition, the Wii Fit game, and newercustomized video games that are geared more towards balance training can be created for use with the computer (Gil-Gómez et al. 2011; Hocine 2011; Lange et al. 2010).

There has been extensive discussion in the literature regardingthe benefits of virtual reality (VR) in rehabilitation (Saposnik et al. 2010; Saposnik \& Levin 2011; Ke et al. 2011; Rahman \& Shaheen 2011). VR refers to a computer-based technology that provides users with computer graphic generated simulated worlds and environments where users can interact and explore whilst receiving multisensory feedback(audio, video, tactile). VR applications can be immersive or nonimmersive depending on the degree of immersion into the computer world and the technology employed.In the former, theuser interacts with the simulated environment through head-mounted displays and other specialized and often expensive devices; whereas in the latter the user is not fully immersed in the environment and cheaper alternative physical sensors are employed. Gaming technologies such as that of the Nintendo Wii are considered as non-immersive VR applications. Such applications allow the user to receive immediate feedback of his/her physical 
actions and movements on the computer screen (registered through the Nintendo Wii and other similar technologies). This is usually presented to the user in the form of either a third person (i.e. seeing a virtual character mirroring his/her movements) or a first person view (i.e. seeing the environment, through the eyes of the virtual character).An additional challenge to consider whenusingcustomized games with the Wii remote controllerfor the purposes of rehabilitation, is that of calibration (Alankus et al. 2010; Annema et al. 2010; Geurts et al 2011; Joo et al. 2010). Calibration refers to manually, or preferably automatically, adjusting the game to the patient's morphology by measuring the abilities of the player, such as the speed, reaction time and range of movement (Geurts et al 2011).

\subsection{Clinical Advantages and Limitations}

As a commercially available, off-the-shelf gaming solution, which is common in many households(Anderson et al. 2010; Butler \& Willett 2010; Crotty et al. 2011; Hurkmans et al. 2011; Lange et al. 2010; Levac et al. 2010), the Nintendo Wii represents a piece of technology, which is familiar to many users of differing ages (Deloitte 2008). In addition it is relatively inexpensive, is well received by therapists and patients alike (Burke et al 2009, Fung et al 2010)and is perceived as a "normal activity" that is not associated with medical treatment or disability(Morelli et al 2010). This may potentially lead to a greater acceptance and willingness to use the technology as part of the rehabilitation process (Annema et al. 2010; Gil-Gómez et al. 2011; Burke et al. 2009; Deutsch et al. 2009; Gerling et al. 2010; Fung et al. 2010; Warland et al. 2012).

The fact that Wii and other gaming systems are considered as safe and enjoyable suggests that patients are more likely to engage in these activities for prolonged periods of time. It has therefore been postulated that use of such systems in rehabilitation has the potential to deliver the intensity and repetition of activity necessary to drive motor recovery( Saposnik et al. 2010). This would be particularly advantageous for home based rehabilitation where lack of adherence to home exercise programs has been a notable issue(Hendrie 2011).

Being primarily a game technology, users can select from a plethora of games and this variety is likely to reduce boredom and further encourage use. However, as they have been targeted for the fitness and leisure industry, not all games are suitable for motor rehabilitation. In fact only a few titles (such as the Wii Sport, Wii fitness) include activities that are appropriate for physicalrehabilitation. The literature (Ballaz and Robert 2011)and specialist web blogs (Scott 2011) have complied a list of titles and corresponding exercises which may be beneficialfor use in rehabilitation. 
While the evidence suggests that off the shelf systems appear to be effective at addressing issues of balance and fitness (Crotty et al 2011, Deutsch et al 2009, Gil-Gomez et al 2011, Morelli et al 2011, Williams et al 2011), there is less evidence in support of the use of the off the shelf Wii for upper limb rehabilitation following stroke, indeed many studies indicate the need for customization for this patient group (Burke et al. 2009; Mouawad \& Doust 2011). Due to problems of reduced range of movement, dexterity, speed and strength that characterizephysical abilities post stroke, stroke survivors with upper limb problems are likely to have difficulty in holding and operating the Wii remote and in engagingwith off the shelf games(Alankus et al. 2010; Joo et al. 2010; Mouawad \& Doust 2011). It is therefore probablethat upper limb rehabilitation following stroke will require customization of games and some form of strapping to attach the Wii remotes to the arm. It should be noted that even amongst patients with the same pathology, a wide range of impairment can be seen and therefore systems will need the ability to be individually calibrated with regard to range of movement, type of exercise,difficulty, and speed.

In addition to providing a motivating and individually calibrated way of rehabilitating patients, as previously described, the ability to bespoke the game sensors means data can be captured and accessed by the therapist and patient for evaluation and monitoring. Nonetheless, while the evidence base for using the Wii in rehabilitation is developing, it remains inconclusive. In part this is due to a lack of studies investigating the efficacy of the device (with 30 studies $(43.5 \%)$ reporting on technical developments alone) but this is also due to methodological weaknesses of manystudies, with poor description resulting in difficulty ascertaining the legitimacy of findings and relevance to the clinical situation. Issues of spontaneous recovery (which is possible for up to 6 months following stroke) and practice effects (when improvement in performance is due to repeated exposure to the outcome measure and not the intervention) may have provided false positive results.In addition, some studies used inappropriate outcome measures, often lacking sensitivity to detect change, thus reducing the likelihood of clinically meaningful results and up take by therapists into practice.

\subsection{Potential of using the Nintendo Wii in rehabilitation}

The literature identifies several advantages, limitations and potential uses for the Nintendo Wii as part of rehabilitation. These are outlined in table 1.

Table 1. Advantages, Limitations and Potential of the Nintendo Wii

\begin{tabular}{|c|c|c|}
\hline Advantages & Limitations & Potential \\
\hline Suitable in its current form & Not suitable for all health & Personalized games can be \\
\hline
\end{tabular}




\begin{tabular}{|c|c|c|}
\hline $\begin{array}{l}\text { for use in rehabilitation of } \\
\text { some health conditions }\end{array}$ & $\begin{array}{l}\text { conditions } \\
\text { Need for calibration for use } \\
\text { with patients with differing } \\
\text { conditions and levels of im- } \\
\text { pairment }\end{array}$ & $\begin{array}{l}\text { designed for specific needs } \\
\text { of patients }\end{array}$ \\
\hline $\begin{array}{l}\text { Relatively low-cost. Acces- } \\
\text { sories can be purchased on } \\
\text { their own }\end{array}$ & $\begin{array}{l}\text { Need to customize to use } \\
\text { accessories on their own }\end{array}$ & \\
\hline $\begin{array}{l}\text { Variety of activities and } \\
\text { games }\end{array}$ & $\begin{array}{l}\text { Unclear whichgames are- } \\
\text { suitable for patient use }\end{array}$ & $\begin{array}{l}\text { Library of games needed } \\
\text { linked to conditions }\end{array}$ \\
\hline \multicolumn{3}{|l|}{$\begin{array}{l}\text { High market penetration in- } \\
\text { to households }\end{array}$} \\
\hline & $\begin{array}{l}\text { Some patients cannot hold } \\
\text { the Wiimote }\end{array}$ & $\begin{array}{l}\text { Can employ armbands to } \\
\text { strap Wiimote to up- } \\
\text { per/lower limbs }\end{array}$ \\
\hline $\begin{array}{l}\text { Not associated with medical } \\
\text { interventions }\end{array}$ & $\begin{array}{l}\text { Game feedback is not pa- } \\
\text { tient-friendly }\end{array}$ & $\begin{array}{l}\text { Can be incorporated into } \\
\text { personalized activities. Pa- } \\
\text { tient useful feedback can be } \\
\text { created }\end{array}$ \\
\hline $\begin{array}{l}\text { Open source libraries to } \\
\text { support connectivity with a } \\
\text { personal computer }\end{array}$ & $\begin{array}{l}\text { Technical knowledge is re- } \\
\text { quired }\end{array}$ & $\begin{array}{l}\text { Partnership opportunities } \\
\text { for engineers and therapists } \\
\text { to work together }\end{array}$ \\
\hline $\begin{array}{l}\text { Enjoyable (effect on moti- } \\
\text { vation and adherence) }\end{array}$ & $\begin{array}{l}\text { No way of monitoring pa- } \\
\text { tient progress and adherence } \\
\text { to rehabilitation regime }\end{array}$ & $\begin{array}{l}\text { Networking capabilities } \\
\text { enabling telerehabilitation } \\
\text { and monitoring }\end{array}$ \\
\hline Few adverse effects & Slow set up & \\
\hline $\begin{array}{l}\text { Gamification of motor reha- } \\
\text { bilitation (patient motiva- } \\
\text { tion) }\end{array}$ & $\begin{array}{l}\text { A few titles suitable for pa- } \\
\text { tient use only }\end{array}$ & $\begin{array}{l}\text { New games customized to } \\
\text { suit the individual needs of } \\
\text { the patient can be created }\end{array}$ \\
\hline $\begin{array}{l}\text { Bluetooth interface allows } \\
\text { connectivity with computers }\end{array}$ & $\begin{array}{l}\text { Bluetooth compatibility is- } \\
\text { sues with different versions } \\
\text { of stack and various ver- } \\
\text { sions of Wiimote }\end{array}$ & \\
\hline $\begin{array}{l}\text { Filtering algorithms can be } \\
\text { used }\end{array}$ & $\begin{array}{l}\text { High level of noise intro- } \\
\text { duced by the sensors' sensi- } \\
\text { tivity }\end{array}$ & \\
\hline \multirow[t]{2}{*}{$\begin{array}{l}\text { Data fusion algorithm pro- } \\
\text { vides a solution to the bias } \\
\text { drift problem }\end{array}$} & $\begin{array}{l}\text { Data fusion algorithm re- } \\
\text { quires the use of IR sensors } \\
\text { to recalibrate yaw over time } \\
\text { in order to compensate the } \\
\text { bias drift. }\end{array}$ & $\begin{array}{l}\text { Accurate } 3 \text { DoF (pitch, roll } \\
\text { and yaw) can be achieved }\end{array}$ \\
\hline & $\begin{array}{l}\text { Evidence base for use in re- } \\
\text { habilitation inconclusive }\end{array}$ & $\begin{array}{l}\text { More research of higher ex- } \\
\text { perimental rigor is required. }\end{array}$ \\
\hline
\end{tabular}


Around 15 million people in England have one or more long-term conditions(Department of Health 2011), a number which is predicted to rise by a third over the next ten years (Department of Health 2011)This, combined with changes in delivery of healthcare (with a greater focus on community care and less hospital based treatment), means that the demand for therapy is set to increase.. Conversely, the numbers of therapists being trained is falling in the UK (CSP 2010a)and there is an acknowledged lack of therapists to supervise exercise in community settings (CSP 2010b). This dichotomy has highlighted the need for patients to be more involved in their care and in particular, being motivated to exercise independently. However, compliance with therapy is notoriously poor(van Dulmen et al. 2008; Engström and Oberg 2005), with a recent study highlighting that within two days of discharge only $79 \%$ of patients werecontinuing with their home exercise program as prescribed(Hendrie 2011). Reasons for lack of compliance are complex and beyond the scope of this paper, however there is evidence that boredom with traditional exercise and a lack of monitoring by therapists are contributing factors(Lewis et al. 2011; Tijou et al. 2010).

Gaming is well received by patients and therapists (Burke et al. 2009; Deutsch et al. 2009, Warland et al 2012) and is embedded into society where it is played by people of all ages (Deloitte 2008). This suggests that the use of VR exercise devices, either as part of a home exercise programor when combined with telerehabilitation, with monitoring abilities, has the potential to help address the important issues of motivation and compliance. This is a particular issue in stroke rehabilitation where greatest recovery is associated with high frequency, high intensity repetitious practice (Langhorne et al. 2009) involving a recommended minimum of 45 minutes daily for 5 days a week(National Institute for Health and Clinical Excellence 2013). Moreover, teletherapy could potentially reduce the need for resource-intensive home and hospital visits and save travel costs(Naylor et al. 2013).

A number of review papers have examined the benefits of virtual reality based systems in stroke and motor rehabilitation in general. However the majority of these, focus on expensive and VR dedicated hardware devices, such as head mounted displays, cyber-gloves, cyber-grasps and haptic devices (i.e. Phantom device, joysticks and force sensors) (Saposnik \& Levin 2011). Although a few studies have investigated the development and use of VR-based interventions and telerehabilitation, all employ one of the aforementioned expensive hardware devices (Huber et al. 2010; Zhang et al. 2008). Only one paper has proposed a Wiibased system offering a telemonitoring facility but this is based on using Nintendo's own network and platform, which is not suitable for holding private and secure patient records (Egglestone et al. 2009). Therefore there is a challenge as well as an opportunity here. The Nintendo Wii could, if customized, presents 
a way to enhance community based stroke rehabilitation of the upper limb by providing a motivating and cost-effective way of exercising that can be remotely monitored and exercises adjusted as required by a physiotherapist.

For this to be implemented a number of key requirements must be fulfilled. The motion capture information (received from the Wii Remote and/or balance board) should be stored on a database, which canmaintain the recorded sessions for each individual patient and beavailable to the therapist for evaluation and monitoring. This would serve as a direct link between the patient and therapist, therefore it couldalso be used as a communication tool (e.g. the therapist couldleave messages for the patient to receive or amend the patient's exercises as $\mathrm{s} / \mathrm{he}$ progresses). As the server relays sensitive information, a set of secure protocols would be necessary to avoid malicious interception from external sources. The use of network sockets would be beneficial for the communication of data between the patient and therapist, as it enables interoperability of the data usage in a cross platform environment (bidirectional inter-process communication flow across the host computer and a remote network) and the ability for the therapist to monitor patient progress both off-line (store-and-forward method) but also in real-time (getting a real-time 3D visualization of the patient's current exercise activities).

Therefore the use of the Nintendo Wii could present a potential solution to community based rehabilitation by providing a novel, motivating way of exercising either as a stand-alone solution or with the addition of teletherapy whenit couldbe remotely monitored and adjusted as required by a physiotherapist.

In addition, Wii-based telerehabilitation interventions support games thatpotentially can foster cooperation and competition amongst community users. Cooperation through gameplay has the potential to increase levels of patient motivation as they couldon one hand feel part of a wider community (of people with a similar condition) where personal experiences and stories could be shared and peersupport facilitated; and on the other, competition wouldprovide an additional incentive for continuing with exercising. Here the research community can make a significant contribution in the form of creating a secure and private online social network, specific to local communities, offering patients with relevant/similar health conditions the opportunity to be socially integrated with the community from their homes.

\subsubsection{Recommendations for Future Studies}

A number of recommendations can be made based on the discussion of the literature.

Technological Recommendations. In addition to the development and testing of teletherapy and activities that can be customized and adapted to individual patient needs (as outlined above), future developments should ensure that systems are 
quick and easy to set up and that frequent feedback which is accurate and motivating is provided (Alankus et al. 2010;). Moreover, new systems should be able to record progress and in addition, they need to be affordable.

Need for Motor Rehabilitation Specific Game Design Guidelines. The customization of a game for rehabilitation purposes is a complex procedure that requires a different approach than traditional game design. The literature suggests that relevant studies utilizing customized games do not always present a specific game design framework or at least some recommendations or guidelines on this matter(Alankus et al. 2010; Annema et al. 2010). Hence it is recommended that a clear game design framework and game design guidelines specific to customized games for motor rehabilitation forms a focus area for future research studies and their accompanied publications.

Research Collaboration. A closer look at the literature presents a picture where most studies have been conducted in isolation with none or little communication between researchers. Each research team uses different commercial games and exercises with different intensity levels. Although a number of customized games and exercise packages have been developed by research teams for the purposes of rehabilitation, most remain inaccessible to the rest of the research and therapy community. Therefore there is a need for the research and therapy communities to work together to produce an online pool where all pilot tested,personalized, adaptable games and exercise packages developed by research teams, are made available with instructions for the wider community to use in practice. This will help in disseminating best practice in the use of such interventions in rehabilitation and in addition, will assist the research community in testing these interventions on a larger scale and thereforewill produce more robust outcomes.

Study Methodology. The issue of experimental rigor is fundamental for helping to promote the uptake of a new therapy into clinical practice, as the drive for evidence based therapy is fundamental. Therefore before a technique can be adopted into clinical practice, it must first be evaluated for efficacy(Campbell 2000)and in addition, it must be evaluated for feasibility and acceptability. In light of this it is imperative that the methodological issues, which characterize much of the literature, are addressed. Study designs need to take into account the potential for spontaneous recovery and practice effects and allow prolonged baseline measures or the use of control groups to account for this. In addition, publications need to more fully describe their methodologies and patient characteristics in order to allow therapists to ascertain the relevance of the study to their patient group. This requires the testing of equipment on the target group involving subjects with the condition under investigation. Furthermore, future studies should consider use of outcome measures which are reliable, validated and sensitive to change. These should address different domains of disability including impairment and functional measures as well as measures of quality of life. 
Compliance. As compliance with an exercise program is key to its success, future studies should examine whether the use of Wii and gaming technology improves compliance with treatment.

Finally, to ensure technical developments are clinically useful and acceptable, the reviewers of this paper strongly recommend engineers, therapists and patients work in collaboration, thereby addressing issues of patient and therapist requirements, issues regarding acceptability and feasibility and an understanding of what is possible technically.

\section{Conclusion}

Through systematic reviewing of the literature, this chapter has outlined the advantages, limitations and the potential uses of the Nintendo Wii as an adjunct in motor rehabilitation. While the Nintendo Wii is not the only commercially available gaming technology where user movement can be tracked, the rationale behind the use and presentation of the Nintendo Wii, arguably lies in its popularity in the home, research and clinical communities. The comparison between the various gaming devices (such asthe Sony Move controller and Microsoft Kinect) is out of the scope of this paper. However several of the benefits, limitations and potentials presented through this paper are applicable to these gaming devices.

The growth of VR systems, in particular interactive gaming has accelerated in the last few years, and the Nintendo Wii stands at the forefront of this development. This chapter has highlightedthe potential of gaming technology within health and social care settings and the importance of collaborative working between engineers, therapists and users of technology. In light of the projected growth in people living with long term health conditions and the resultant effect on health and social care resources, the need for evidence based, innovations which promote self-management are required to meet these challenges.

\section{References}

Alankus G, Lazar A, May M, Kelleher C (2010) Towards customizable games for stroke rehabilitation. Proc. 28th Int. Conf. Hum. factors Comput. Syst. - CHI '10. ACM Press, New York, New York, USA, p 2113 
Alankus G, Proffitt R (2010) Stroke therapy through motion-based games: a case study. 12th Int. ACM SIGACCESS Conf. Comput. Access. (ASSETS ’10). ACM, pp 219-226

Anderson F, Annett M, Bischof WF (2010) Lean on Wii: physical rehabilitation with virtual reality Wii peripherals. Stud Health Technol Inform 154:229-34.

Annema J, Verstraete M, Abeele V Vanden, Desmet S, Geerts D, Leuven IKU (2010) Videogames in therapy: a therapist's perspective. Proc. 3rd 3rd Int. Conf. Fun Games. ACM, pp 94-98

Attygalle S, Duff M (2008) Low-cost, at-home assessment system with Wii Remote based motion capture. Virtual Rehabil 2008 168-174.

Bainbridge E, Bevans S, Keeley B, Oriel K (2011) The Effects of the Nintendo Wii Fit on CommunityDwelling Older Adults with Perceived Balance Deficits: A Pilot Study. Phys Occup Ther Geriatr 29:126-135. doi: 10.3109/02703181.2011.569053

Ballaz L, Robert M (2011) Active video games and children with cerebral palsy: the future of rehabilitation? Virtual Rehabil. Int. Conf. pp 1-2

Bateni H (2012) Changes in balance in older adults based on use of physical therapy vs the Wii Fit gaming system: a preliminary study. Physiotherapy 98:211-6. doi: 10.1016/j.physio.2011.02.004

Brochard S, Robertson J, Medee B, Remy-Neris O (2010) What's new in new technologies for upper extremity rehabilitation? Curr Opin Neurol 23:683-687.

Burke J, McNeill M, Charles D, Morrow P, Crosbie J, McDonough S (2009) Serious Games for Upper Limb Rehabilitation Following Stroke. 2009 Conf Games Virtual Worlds Serious Appl 103110. doi: 10.1109/VS-GAMES.2009.17

Burke J, Morrow P, McNeill M, McDonough S, Charles D (2008) Vision Based Games for UpperLimb Stroke Rehabilitation. 2008 Int Mach Vis Image Process Conf 159-164. doi: 10.1109/IMVIP 2008.16

Butler DP, Willett K (2010) Wii-habilitation: is there a role in trauma? Injury 41:883-5. doi: 10.1016/j.injury.2010.03.024

Campbell M (2000) Framework for design and evaluation of complex interventions to improve health. BMJ 321:694-696.

Clark R a, Bryant AL, Pua Y, McCrory P, Bennell K, Hunt M (2010) Validity and reliability of the Nintendo Wii Balance Board for assessment of standing balance. Gait Posture 31:307-10. doi: 10.1016/j.gaitpost.2009.11.012

Crotty M, Laver MK, Quinn S, Ratcliffe PJ, George S, Craig AP, Participants A (2011) Is use of the Nintendo Wii Fit in physiotherapy as effective as conventional physiotherapy training for hospitalised older adults? A pilot randomised controlled trial. Virtual Rehabil. Int. Conf. pp $2-$ 
CSP (2010a) Submission to the Centre for Workforce Intelligence on the future physiotherapy workforce. London, UK

CSP (2010b) Moving on: A vision for community based physiotherapy after stroke in England. 1-21.

Deloitte (2008) The Retail Review Retail Survey. 1-23.

Department of Health (2011) Ten things you need to know about long term conditions.

Deutsch JE, Robbins D, Morrison J, Guarrera Bowlby P (2009) Wii-based compared to standard of care balance and mobility rehabilitation for two individuals post-stroke. 2009 Virtual Rehabil Int Conf 117-120. doi: 10.1109/ICVR.2009.5174216

Van Dulmen S, Sluijs E, van Dijk L, de Ridder D, Heerdink R, Bensing J (2008) Furthering patient adherence: a position paper of the international expert forum on patient adherence based on an internet forum discussion. BMC Health Serv Res 8:47. doi: 10.1186/1472-6963-8-47

Egglestone SR, Axelrod L, Nind T, Turk R, Wilkinson A, Burridge J, Fitzpatrick G, Mawson S, Robertson Z, Hughes AM, Ng KH, Pearson W, Shublaq N, Probert-Smith P, Rickets I, Rodden $\mathrm{T}$ (2009) A design framework for a home-based stroke rehabilitation system: Identifying the key components. Proc 3d Int ICST Conf Pervasive Comput Technol Healthc. doi: 10.4108/ICST.PERVASIVEHEALTH2009.6049

Engström LO, Oberg B (2005) Patient adherence in an individualized rehabilitation programme: a clinical follow-up. Scand J Public Health 33:11-8. doi: 10.1080/14034940410028299

Featherstone R (1987) Robot Dynamics Algorithms. Springer

Fung V, Ho A, Shaffer J, Chung E, Gomez M (2012) Use of Nintendo Wii Fit ${ }^{\mathrm{TM}}$ in the rehabilitation of outpatients following total knee replacement: a preliminary randomised controlled trial. Physiotherapy 98:183-8. doi: 10.1016/j.physio.2012.04.001

Fung V, Park H, Ho A, Shaffer J, Chan E, Gomez M (2010) The Utility of a Video Game System in Rehabilitation of Burn and Nonburn Patients: A Survey Among Occupational Therapy and Physiotherapy Practitioners. J Burn Care Res Burn Care Res 31:768-775.

Galego B, Simone L (2007) Leveraging online virtual worlds for upper extremity Rehabilitation. 2007 IEEE 33rd Annu. Northeast Bioeng. Conf. Ieee, pp 267-268

Gerling K, Schild J, Masuch M (2010) Exergame design for elderly users: the case study of SilverBalance. Int. Conf. Adv. Comput. Entertain. Technol. Taiwan, pp 66-69

Geurts L, Abeele V Vanden (2011) Digital games for physical therapy: fulfilling the need for calibration and adaptation. Proc. fifth Int. Conf. Tangible, Embed. embodied Interact. ACM, New York, pp 117-124

Gil-Gómez J-A, Lloréns R, Alcañiz M, Colomer C (2011) Effectiveness of a Wii balance board-based system $(\mathrm{eBaViR})$ for balance rehabilitation: a pilot randomized clinical trial in patients with acquired brain injury. J Neuroeng Rehabil 8:30. doi: 10.1186/1743-0003-8-30 
gl.tter (2013) WiiYourself! - A native C++ library. http://wiiyourself.gl.tter.org/. Accessed 15 Mar 2013

Göbel S, Hardy S, Wendel V (2010) Serious games for health: personalized exergames. Proc. Int. Conf. Multimed. ACM, New York, pp 1663-1666

Henderson A, Korner-Bitensky N, Levin M (2007) Virtual reality in stroke rehabilitation: a systematic review of its effectiveness for upper limb motor recovery. Top Stroke Rehabil 14:52-61. doi: $10.1310 / \operatorname{tsr} 1402-52$

Hendrie W (2011) The dog ate my slippers. Synapse 25:

Hocine N (2011) Therapeutic Games ' Difficulty Adaptation : An Approach Based on Player's Ability and Motivation. 16 th Int. Conf. Comput. Games. IEEE, pp 257-261

Huber M, Rabin B, Docan C, Burdea GC, AbdelBaky M, Golomb MR (2010) Feasibility of modified remotely monitored in-home gaming technology for improving hand function in adolescents with cerebral palsy. IEEE Trans Inf Technol Biomed 14:526-34. doi: 10.1109/TITB.2009.2038995

Hurkmans HL, Ribbers GM, Streur-Kranenburg MF, Stam HJ, van den Berg-Emons RJ (2011) Energy expenditure in chronic stroke patients playing Wii Sports: a pilot study. J Neuroeng Rehabil 8:38. doi: 10.1186/1743-0003-8-38

Jovanov E, Hanish N, Courson V, Stidham J, Stinson H, Webb C, Denny K (2009) Avatar - a multisensory system for real time body position monitoring. Conf Proc IEEE Eng Med Biol Soc 2009:2462-5. doi: 10.1109/IEMBS.2009.5334774

Jung Y, Li K, Janissa N (2009) Games for a better life: effects of playing Wii games on the well-being of seniors in a long-term care facility. Proc. Sixth Australas. Conf. Interact. Entertain. ACM, New York, pp 0-5

Kalman R (1960) A new approach to linear filtering and prediction problems. J Basic Eng 82:33-45.

Kennedy MW, Schmiedeler JP, Crowell CR, Villano M, Striegel AD, Kuitse J (2011) Enhanced feedback in balance rehabilitation using the Nintendo Wii Balance Board. 2011 IEEE 13th Int Conf e-Health Networking, Appl Serv 162-168. doi: 10.1109/HEALTH.2011.6026735

Kenner C (2010) GlovePIE - A free Programmable Input Emulator. http://glovepie.org/. Accessed 15 Mar 2013

Kho ME, Damluji A, Zanni JM, Needham DM (2012) Feasibility and observed safety of interactive video games for physical rehabilitation in the intensive care unit: a case series. J Crit Care 27:219.e1-6. doi: 10.1016/j.jcrc.2011.08.017

Kwok BC, Mamun K, Chandran M, Wong CH (2011) Evaluation of the Frails' Fall Efficacy by Comparing Treatments (EFFECT) on reducing fall and fear of fall in moderately frail older adults: study protocol for a randomised control trial. Trials 12:155. doi: 10.1186/1745-6215-12155 
Laforest M (2010) wiiuse - A Cross-platform C library. http://www.wiiuse.net/docs/. Accessed 15 Mar 2013

Lange B, Chang C-Y, Suma E, Newman B, Rizzo AS, Bolas M (2011) Development and evaluation of low cost game-based balance rehabilitation tool using the Microsoft Kinect sensor. Conf Proc IEEE Eng Med Biol Soc 2011:1831-4. doi: 10.1109/IEMBS.2011.6090521

Lange B, Flynn S, Proffitt R, Chang C-Y, Rizzo AS (2010) Development of an interactive game-based rehabilitation tool for dynamic balance training. Top Stroke Rehabil 17:345-52. doi: $10.1310 / \operatorname{tsr} 1705-345$

Langhorne P, Coupar F, Pollock A (2009) Motor recovery after stroke: a systematic review. Lancet Neurol 8:741-54. doi: 10.1016/S1474-4422(09)70150-4

Laver K, George S, Thomas S, Deutsch J, Crotty M (2011) Virtual reality for stroke rehabilitation (Review). Cochrane Collab 1-72.

Laviola J, Marks J, L R, Marksplaystationsonycom R (2010) An Introduction to 3D Spatial Interaction with Video Game Motion Controllers. ACM SIGGRAPH 2010 Courses. New York, p 78

Leder R, Azcarate G (2008) Nintendo Wii remote for computer simulated arm and wrist therapy in stroke survivors with upper extremity hemipariesis. Virtual Rehabil. Vancouver, Canada, p 47

Lehrer N, Chen Y, Duff M, Wolf S, Rikakis T (2011) Exploring the bases for a mixed reality stroke rehabilitation system, part ii: Design of interactive feedback for upper limb rehabilitation. $\mathrm{J}$ Neuroeng Rehabil 8:54.

Levac D, Pierrynowski MR, Canestraro M, Gurr L, Leonard L, Neeley C (2010) Exploring children's movement characteristics during virtual reality video game play. Hum Mov Sci 29:1023-38. doi: 10.1016/j.humov.2010.06.006

Lewis GN, Woods C, Rosie J a, McPherson KM (2011) Virtual reality games for rehabilitation of people with stroke: perspectives from the users. Disabil Rehabil Assist Technol 6:453-63. doi: $10.3109 / 17483107.2011 .574310$

Ma M, Bechkoum K (2008) Serious games for movement therapy after stroke. 2008 IEEE Int. Conf. Syst. Man Cybern. Ieee, Singapore, pp 1872-1877

Madgwick S (2010) An efficient orientation filter for inertial and inertial/magnetic sensor arrays. University of Bristol

Manlapaz DG, Silverio L a., Navarro J a., Ang MF, Regacho M, Canaberal K a., Dela Cruz RB (2010) Effectiveness of Using Nintendo Wii in Rehabilitation of Chronic Stroke Patients with Upper Limb Hemiparesis. Hong Kong Physiother J 28:25. doi: 10.1016/j.hkpj.2010.11.014

Martin-Moreno J, Ruiz-Fernandez D, Soriano-Paya A, Jesus Berenguer-Miralles V (2008) Monitoring 3D movements for the rehabilitation of joints in physiotherapy. Conf. Proc. IEEE Eng. Med. Biol. Soc. pp 4836-9 
Matamoros M, Negrete M (2010) Nintendo WII remote and Nunchuck as a wireless data subsystem for digital acquisition of analog physiologic data relevant to motor rehabilitation after stroke; part II. Panam. Heal. Care Exch. IEEE, Lima, pp 1-3

Meditch JS (1973) A Survey of Data Smoothing for Linear and Nonlinear. Automatica 9:151-162.

Milosevic M, Jovanov E (2011) A real-time control of multiple Avatars using Wii remotes and Avatar system. 2011 IEEE 43rd Southeast. Symp. Syst. Theory. Ieee, pp 139-142

Morelli T, Foley J, Folmer E (2010) Vi-bowling: a tactile spatial exergame for individuals with visual impairments. Proc. 12th Int. ACM SIGACCESS Conf. Comput. Access. ACM, New York, pp 179-186

Morelli T, Foley J, Lieberman L, Folmer E (2011) Pet-N-Punch: upper body tactile/audio exergame to engage children with visual impairments into physical activity. Proc. Graph. Interface. Canadian Human-Computer Communications Societ, Ontario, pp 223-230

Mouawad M, Doust C (2011) Wii-based movement therapy to promote improved upper extremity function post-stroke: a pilot study. J Rehabil Med 43:527-533.

National Institute for Health and Clinical Excellence (2013) Stroke rehabilitation: long-term rehabilitation after stroke. $1-45$.

Naylor C, Imison C, Addicott R, Buck D, Goodwin N, Harrison T, Ross S, Sonola L, Tian Y, Curry N (2013) Transforming our health care system Ten priorities for commissioners. 1-18.

Nitz JC, Kuys S, Isles R, Fu S (2010) Is the Wii Fit a new-generation tool for improving balance, health and well-being? A pilot study. Climacteric 13:487-91. doi: $10.3109 / 13697130903395193$

Oppenheim H (2010) WiiEMG: A real-time environment for control of the Wii with surface electromyography. IEEE Int. Symp. Circuits Syst. IEEE, Paris, pp 957-960

Peek B (2010) Managed Library for Nintendo's Wiimote. http://wiimotelib.codeplex.com/. Accessed 15 Mar 2013

Plow M, Finlayson M (2012) A Qualitative Study Exploring the Usability of Nintendo Wii Fit among Persons with Multiple Sclerosis. Occup Ther Int. doi: 10.1002/oti.1345

Prashun P, Hadley G, Gatzidis C, Swain I (2010) Investigating the Trend of Virtual Reality-Based Stroke Rehabilitation Systems. 2010 14th Int Conf Inf Vis 641-647. doi: 10.1109/IV.2010.93

Rahman S, Shaheen A (2011) Virtual reality use in motor rehabilitation of neurological disorders: A systematic review. Middle-East J Sci Res 7:63-70.

Robinson J, Schaik P Van (2011) User-acceptance and flow in two gaming platforms used for exercise. Virtual Rehabil. Int. Conf. Ieee, Zurich, pp 26-27

Saposnik G, Levin M (2011) Virtual reality in stroke rehabilitation: a meta-analysis and implications for clinicians. Stroke 42:1380-6. doi: 10.1161/STROKEAHA.110.605451 
Saposnik G, Teasell R, Mamdani M, Hall J (2010a) Effectiveness of Virtual Reality Using Wii Gaming Technology in Stroke Rehabilitation A Pilot Randomized Clinical Trial and Proof of Principle. Stroke 41:1477-1484.

Saposnik G, Teasell R, Mamdani M, Hall J, McIlroy W, Cheung D, Thorpe KE, Cohen LG, Bayley M (2010b) Effectiveness of virtual reality using Wii gaming technology in stroke rehabilitation: a pilot randomized clinical trial and proof of principle. Stroke 41:1477-84. doi: 10.1161/STROKEAHA.110.584979

Scherfgen D, Herpers R (2009) 3D tracking using multiple Nintendo Wii Remotes: a simple consumer hardware tracking approach. Proc. 2009 Conf. Futur. Play. ACM, Vancouver, pp 31-32

Scott R (2011) Wiihabilitation. http://www.wiihabilitation.co.uk/. Accessed 15 Mar 2013

Seifert K, Camacho O (2007) Implementing Positioning Algorithms Using Accelerometers. 1-13.

Smith L (2011) CWiid - A collection of Linux tools written in C for interfacing to the Nintendo Wiimote. http://abstrakraft.org/cwiid/. Accessed 15 Mar 2013

Sugarman H, Weisel-Eichler A, Burstin A, Brown R (2009) Use of the Wii Fit system for the treatment of balance problems in the elderly: A feasibility study. 2009 Virtual Rehabil. Int. Conf. Ieee, pp $111-116$

Tijou I, Yardley L, Sedikides C, Bizo L (2010) Understanding adherence to physiotherapy: findings from an experimental simulation and an observational clinical study. Psychol Health 25:23147. doi: $10.1080 / 08870440802372431$

Tsekleves E, Skordoulis D, Paraskevopoulos I, Kilbride C (2011) Wii Your Health: A Low-Cost Wireless System for Home Rehabilitation after Stroke using Wii Remotes with its Expansions and Blender. Proc Biomed Eng. doi: http://dx.doi.org/10.2316/P.2011.723-058

Warland A, Kilbride C, Tsekleves E, Skordoulis D, Paraskevopoulos I (2012) ReWiiRe (Research in Wii Rehabilitation): Personalised, involvement in the development of a After, rehabilitation system for arm re-education Stroke. UK Stroke Forum. Harrogate, UK, p 27

Van Wieringen M, Eklund J (2008) Real-time signal processing of accelerometer data for wearable medical patient monitoring devices. Conf. Proc. IEEE Eng. Med. Biol. Soc. pp 2397-400

Williams B, Doherty NL, Bender A, Mattox H, Tibbs JR (2011) Study Supporting the Use of the Wii in Occupational Therapy for the Well Elderly. Occup Ther Heal 25:131-139. doi: $10.3109 / 07380577$

Williams M a, Soiza RL, Jenkinson AM, Stewart A (2010) EXercising with Computers in Later Life (EXCELL) - pilot and feasibility study of the acceptability of the Nintendo® WiiFit in community-dwelling fallers. BMC Res Notes 3:238. doi: 10.1186/1756-0500-3-238

Williamson B, Wingrave C, LaViola J (2010) Realnav: Exploring natural user interfaces for locomotion in video games. IEEE Symp. 3D User Interfaces. IEEE, pp 3-10 
Wilson PH, Duckworth J, Mumford N, Eldridge R, Guglielmetti M, Thomas P, Shum D, Rudolph H (2007) A virtual tabletop workspace for the assessment of upper limb function in Traumatic Brain Injury (TBI). 2007 Virtual Rehabil 14-19. doi: 10.1109/ICVR.2007.4362122

Wuang Y-P, Chiang C-S, Su C-Y, Wang C-C (2011) Effectiveness of virtual reality using Wii gaming technology in children with Down syndrome. Res Dev Disabil 32:312-21. doi: 10.1016/j.ridd.2010.10.002

Yong Joo L, Soon Yin T, Xu D, Thia E, Pei Fen C, Kuah CWK, Kong K-H (2010) A feasibility study using interactive commercial off-the-shelf computer gaming in upper limb rehabilitation in patients after stroke. J Rehabil Med 42:437-41. doi: 10.2340/16501977-0528

Zhang S, Hu H, Zhou H (2008) An interactive Internet-based system for tracking upper limb motion in home-based rehabilitation. Med Biol Eng Comput 46:241-9. doi: 10.1007/s11517-007-0295-6 